Supporting information

\title{
Life Cycle Assessment of an Enzymatic Ibuprofen Production Process with Automatic Recycling and Purification
}

\author{
Fabio Grimaldi ${ }^{1}$, Nam Nghiep Tran ${ }^{2,3^{*}}$, Mohammad Mohsen Sarafraz ${ }^{2,4}$, Paola Lettieri ${ }^{1 *}$, Olivia M. Morales \\ Gonzalez ${ }^{5}$, Volker Hessel ${ }^{2.6 *}$ \\ ${ }^{1}$ University College London, London, United Kingdom \\ ${ }^{2}$ School of Chemical Engineering and Advanced Materials, University of Adelaide, Australia \\ ${ }^{3}$ School of Chemical Engineering, Can Tho University, Vietnam \\ ${ }^{4}$ School of Engineering, Deakin University, Australia \\ ${ }^{5}$ Department of Chemical Engineering and Chemistry, Eindhoven University of Technology \\ ${ }^{6}$ School of Engineering, University of Warwick, United Kingdom \\ *Email: volker.hessel@adelaide.edu.au \\ namnghiep.tran@adelaide.edu.au \\ p.lettieri@ucl.ac.uk
}

A. LCA Methodology

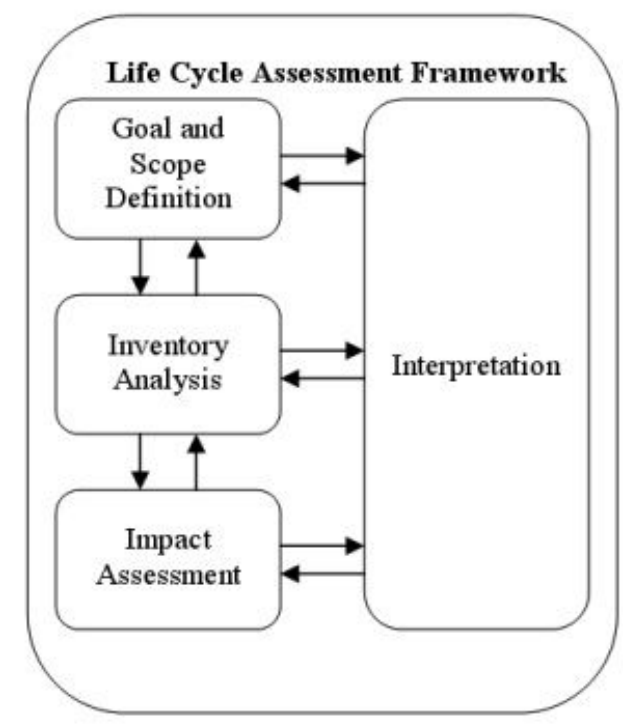

Figure S1. Life Cycle Assessment Framework

\section{B. Data gaps, allocation, and uncertainty of the LCA study}

Data gaps: when no datasets were available to describe the life cycle of precursors, we followed a retro-synthesis approach, approximating the life cycle impacts of such precursor to the impact of the chemicals that ladder up to the precursor. The screenshots (Figure S1 to S3) presented below contain information on the above. IL is modelled in a separate plan in $\mathrm{GaBi}$ and is reported as a separate screenshot (Figure S4).

Allocation: since the synthesis of ibuprofen generates almost no by-products, the impact of the upstream system is allocated to ibuprofen and we followed a cut-off method.

Uncertainty: depending on the simulation data and the availability of existing LCA datasets in $\mathrm{GaBi}$, we have used different levels of uncertainty in the LCI (low, medium-high $+-5 / 10 / 15 \%$ ). This was not reported originally in the manuscript for sake of brevity but it is now reported below. 
Table S1. Uncertainty error associated with LCI data for modified Bogdan synthetic route

\begin{tabular}{lll}
\hline $\begin{array}{l}\text { LCI: Modified Bogdan (Enzymatic } \\
\text { catalyst) }\end{array}$ & $\begin{array}{l}\text { Level of } \\
\text { uncertainty }\end{array}$ & $\%$ \\
\hline HE Heat production & LOW & $5 \%$ \\
SC1 Hazardous waste & HIGH & $15 \%$ \\
R1 Heat production & LOW & $5 \%$ \\
R1 Isobutylbenzene & MEDIUM & $10 \%$ \\
R1 Propionic acid & MEDIUM & $10 \%$ \\
R1 Trifflic acid & HIGH & $15 \%$ \\
R2 Trifflic acid & HIGH & $15 \%$ \\
SC2 Hazardous waste & HIGH & $15 \%$ \\
R2 Heat production & LOW & $5 \%$ \\
R2 Iodobenzene diacetate & HIGH & $15 \%$ \\
R2 Methanol & MEDIUM & $10 \%$ \\
R2 Trimethyl orthoformate & HIGH & $15 \%$ \\
SC3 Hazardous waste & HIGH & $15 \%$ \\
R3 Heat production & LOW & $5 \%$ \\
R3 Ionic liquid & HIGH & $5 \%$ \\
R3 Phosphate buffer solution & MEDIUM \\
R3 Water & LOW & $10 \%$ \\
SC1 Heat production & LOW & $5 \%$ \\
SC2 Heat production & L & $5 \%$ \\
\hline
\end{tabular}


Table S2. Uncertainty error associated with LCI data for the Bogdan synthetic route

\begin{tabular}{lll}
\hline LCI: Bogdan & $\begin{array}{l}\text { Level of } \\
\text { uncertainty }\end{array}$ & \% \\
\hline HE Heat production & HIGH & $0 \%$ \\
SC1 Hazardous waste & LOW & $5 \%$ \\
R1 Heat production & HIGH & $15 \%$ \\
R1 Isobutylbenzene & LOW & $5 \%$ \\
R1 Propionic acid & MEDIUM & $10 \%$ \\
R1 Trifflic acid & MEDIUM & $10 \%$ \\
R2 Trifflic acid & HIGH & $15 \%$ \\
SC2 Hazardous waste & HIGH & $15 \%$ \\
R2 Heat production & HIGH & $15 \%$ \\
R2 Iodobenzene diacetate & LOW & $5 \%$ \\
R2 Methanol & HIGH & $15 \%$ \\
R2 Trimethyl orthoformate & MEDIUM & $10 \%$ \\
SC3 Hazardous waste & HIGH & $15 \%$ \\
R3 Heat production & HIGH & $15 \%$ \\
R3 Methanol & MOW & $10 \%$ \\
R3 Potassium hydroxide & MEDIUM \\
R3 Water & MEDIUM \\
\hline SC3 Heat production & & $10 \%$ \\
\hline
\end{tabular}


Table S3. Uncertainty error associated with LCI data for the BHC synthetic route

\begin{tabular}{lll}
\hline LCI: BHC & $\begin{array}{l}\text { Level of } \\
\text { uncertainty }\end{array}$ & \% \\
\hline HE Heat production & LOW & $5 \%$ \\
R1 Acetic anhydride & HIGH & $15 \%$ \\
SC1 Hazardous waste & HIGH & $15 \%$ \\
R1 Heat production & LOW & $5 \%$ \\
R1 Hydrofluoric acid & MEDIUM & $10 \%$ \\
R1 Isobutylbenzene & HIGH & $15 \%$ \\
SC2 Hazardous waste & HIGH & $15 \%$ \\
R2 Heat production & LOW & $5 \%$ \\
R2 Hydrogen & MEDIUM & $10 \%$ \\
R2 Raney Nickel & MEDIUM & $10 \%$ \\
R3 Carbon monoxide & MEDIUM & $10 \%$ \\
SC3 Hazardous waste & HIGH & $15 \%$ \\
R3 Heat production & LOW & $5 \%$ \\
R3 Palladium(II) chloride & HIGH & $15 \%$ \\
R3 Triphenylphosphine & LOWH & $5 \%$ \\
SC1 Heat production & & $5 \%$ \\
SC2 Heat production & LOW & \\
SC3 Heat production & & $5 \%$ \\
\hline & & $5 \%$ \\
\hline
\end{tabular}


Table S4: LCIA cumulative error per impact categories and scenarios

\begin{tabular}{lllll}
\hline & $\begin{array}{l}\text { LCIA: Modified } \\
\text { Bogdan (Enzymatic } \\
\text { catalyst) } \mathbf{1 0 0} \%\end{array}$ & $\begin{array}{l}\text { LCIA: Modified } \\
\text { Bogdan (Enzymatic } \\
\text { catalyst) }\end{array}$ & $\begin{array}{l}\text { LC } \% \\
\text { Bogdan }\end{array}$ & $\begin{array}{l}\text { LCIA: } \\
\text { BHC }\end{array}$ \\
LCIA: Cumulative error & & & & \\
\hline A & $14 \%$ & $15 \%$ & $13 \%$ & $14 \%$ \\
CC & $15 \%$ & $15 \%$ & $14 \%$ & $13 \%$ \\
EcoTOX & $15 \%$ & $15 \%$ & $14 \%$ & $12 \%$ \\
E fw & $15 \%$ & $15 \%$ & $14 \%$ & $13 \%$ \\
E mw & $15 \%$ & $15 \%$ & $13 \%$ & $12 \%$ \\
E t & $14 \%$ & $15 \%$ & $13 \%$ & $12 \%$ \\
HT c & $15 \%$ & $15 \%$ & $15 \%$ & $13 \%$ \\
HT non-c & $15 \%$ & $15 \%$ & $14 \%$ & $12 \%$ \\
IR & $14 \%$ & $15 \%$ & $13 \%$ & $11 \%$ \\
OD & $15 \%$ & $15 \%$ & $13 \%$ & $12 \%$ \\
PM & $14 \%$ & $15 \%$ & $13 \%$ & $13 \%$ \\
POF & $14 \%$ & $15 \%$ & $13 \%$ \\
\hline RD water & $14 \%$ & $15 \%$ & $13 \%$ \\
\hline
\end{tabular}




\section{Life Cycle Impact Assessment (LCIA)}

Table S5 Impact categories used for the LCIA and description: the impact categories are selected from the LCIA method 'ILCD/PEF recommendation 1.09'.

\begin{tabular}{|c|c|c|c|}
\hline Impact category & Abbr. & Description & Unit \\
\hline Acidification & $\bar{A}$ & $\begin{array}{l}\text { It is mainly caused by air emissions of } \\
\mathrm{NH} 3, \mathrm{NO} 2 \text { and } \mathrm{SOx} \text {. }\end{array}$ & [mol H $\mathrm{H}^{+}$eq.] \\
\hline Climate change & $\mathrm{CC}$ & $\begin{array}{l}\text { Contributions of the greenhouse gases } \\
\text { to global warming and climate change }\end{array}$ & {$\left[\mathrm{kg} \mathrm{CO}_{2}\right.$ eq. $]$} \\
\hline $\begin{array}{l}\text { Ecotoxicity } \\
\text { freshwater midpoint }\end{array}$ & EcoTOX & $\begin{array}{l}\text { Toxic effect on aquatic freshwater } \\
\text { species in the water ecosystems. }\end{array}$ & [CTUe] \\
\hline $\begin{array}{l}\text { Eutrophication } \\
\text { freshwater midpoint }\end{array}$ & E fw & $\begin{array}{l}\text { Eutrophication effects in the freshwater } \\
\text { compartment. }\end{array}$ & [kg P eq.] \\
\hline $\begin{array}{l}\text { Eutrophication } \\
\text { marine midpoint }\end{array}$ & E mw & $\begin{array}{l}\text { Eutrophication effects in the marine } \\
\text { compartment. }\end{array}$ & [kg N eq.] \\
\hline $\begin{array}{l}\text { Eutrophication } \\
\text { terrestrial midpoint }\end{array}$ & Et & $\begin{array}{l}\text { Eutrophication effects in the terrestrial } \\
\text { compartment. }\end{array}$ & [mol $\mathrm{N}$ eq.] \\
\hline $\begin{array}{l}\text { Human toxicity } \\
\text { midpoint, cancer } \\
\text { effects }\end{array}$ & HT c & $\begin{array}{l}\text { Toxic effect on humans referring to } \\
\text { potential cancer effects. }\end{array}$ & [CTUh] \\
\hline $\begin{array}{l}\text { Human toxicity } \\
\text { midpoint, non- } \\
\text { cancer effects }\end{array}$ & HT non-c & $\begin{array}{l}\text { Toxic effect on humans referring to } \\
\text { potential non-cancer effects. }\end{array}$ & [CTUh] \\
\hline Ozone depletion & OD & $\begin{array}{l}\text { Depletion of the ozone layer at the } \\
\text { stratosphere level. }\end{array}$ & [kg CFC-11 eq.] \\
\hline Ionising radiation & IR & $\begin{array}{l}\text { Human exposure to ionizing radiation } \\
\text { with potential alterations in the DNA }\end{array}$ & [kBq U235 eq.] \\
\hline Particulate matter & PM & $\begin{array}{l}\text { Direct and indirect contribution to } \\
\text { particulate matter formation }\end{array}$ & [kg PM2.5 eq.] \\
\hline $\begin{array}{l}\text { Photochemical } \\
\text { ozone formation } \\
\text { midpoint }\end{array}$ & POF & $\begin{array}{l}\text { Contributions of VOC (volatile organic } \\
\text { compounds) and non-VOC to the } \\
\text { formation of ozone at troposphere } \\
\text { level. }\end{array}$ & $\begin{array}{l}{[\mathrm{kg} \text { NMVOC }} \\
\text { eq.] }\end{array}$ \\
\hline $\begin{array}{l}\text { Resource depletion, } \\
\text { water }\end{array}$ & RD water & Water resource depletion. & {$\left[\mathrm{m}^{3}\right]$} \\
\hline $\begin{array}{l}\text { Resource depletion, } \\
\text { minerals, fossils } \\
\text { and renewables }\end{array}$ & $\begin{array}{l}\mathrm{RD} m, \mathrm{f} \\
\text { ren }\end{array}$ & $\begin{array}{l}\text { Depletion of mineral and fossil } \\
\text { resources. }\end{array}$ & [kg Sb eq.] \\
\hline
\end{tabular}




\section{GaBi approaches for the three simulated scenarios}

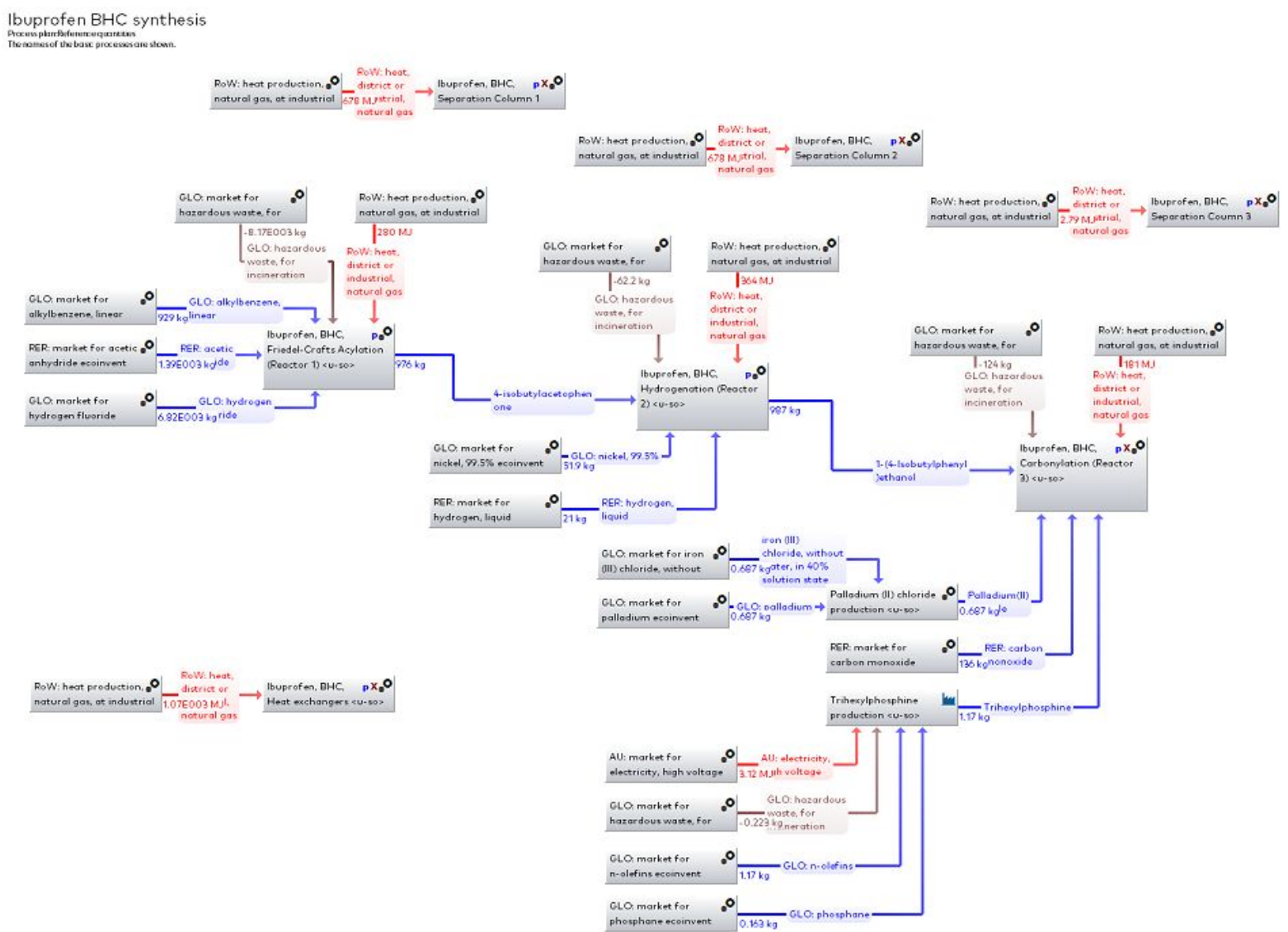

Figure S2. LCA approach for the BHC synthetic route developed through GaBi 


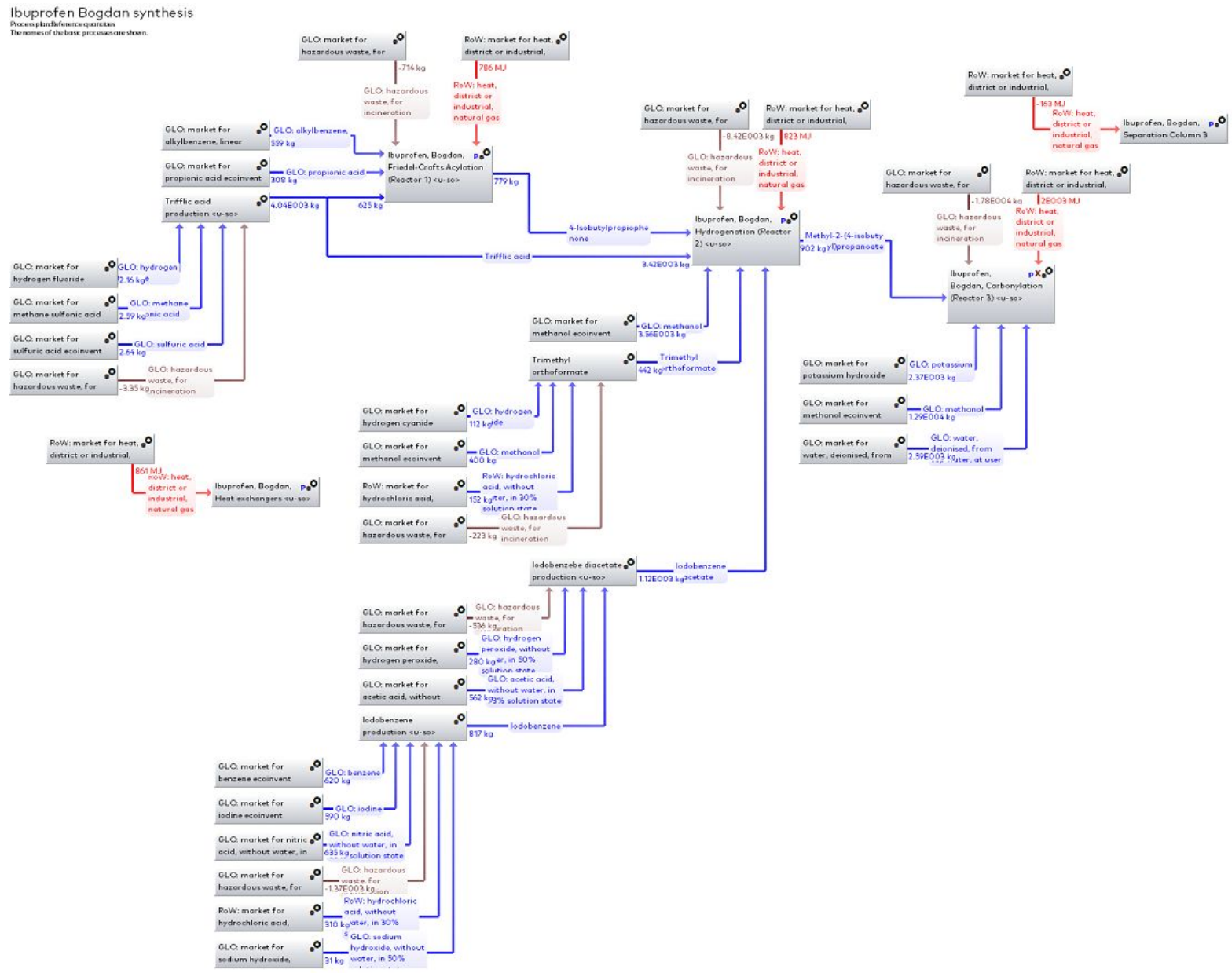

Figure S3. LCA approach for the Bogdan synthetic route developed through GaBi 


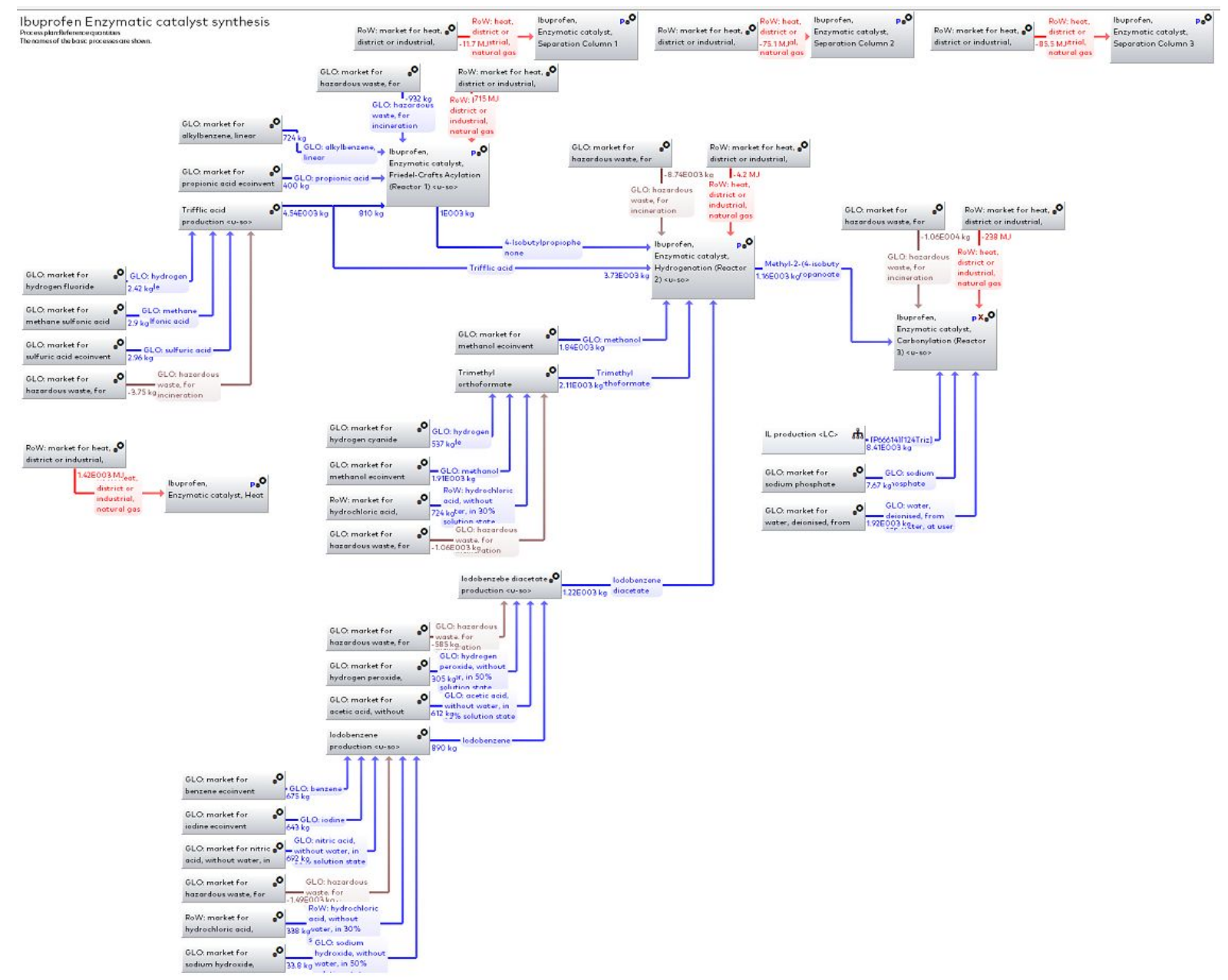

Figure S4. LCA approach for the modified Bogdan synthetic route developed through GaBi 


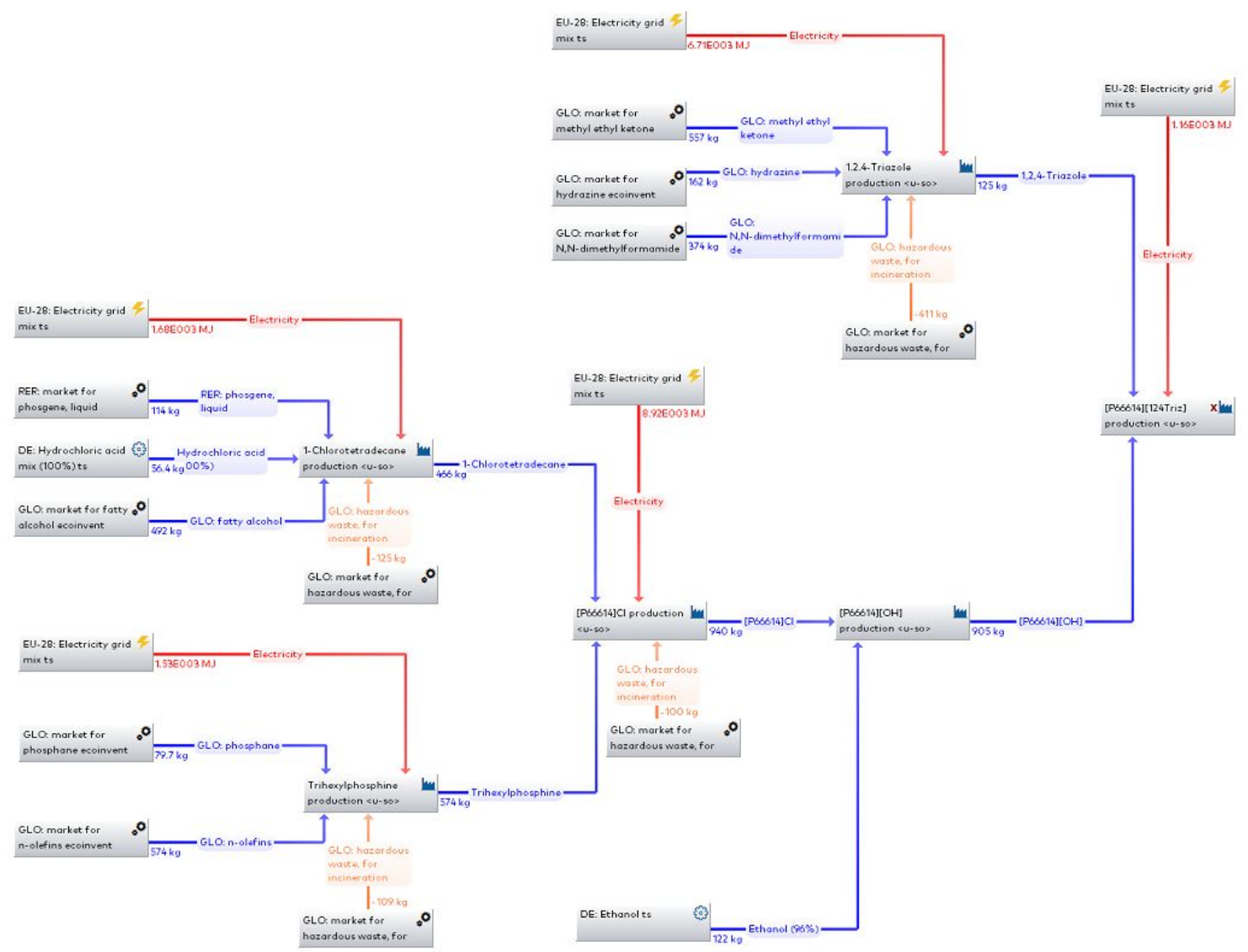

Figure S5. LCA approach for the ionic liquid developed through $\mathrm{GaBi}$ 


\section{E. Aspen simulation results}

E1. BHC synthetic route

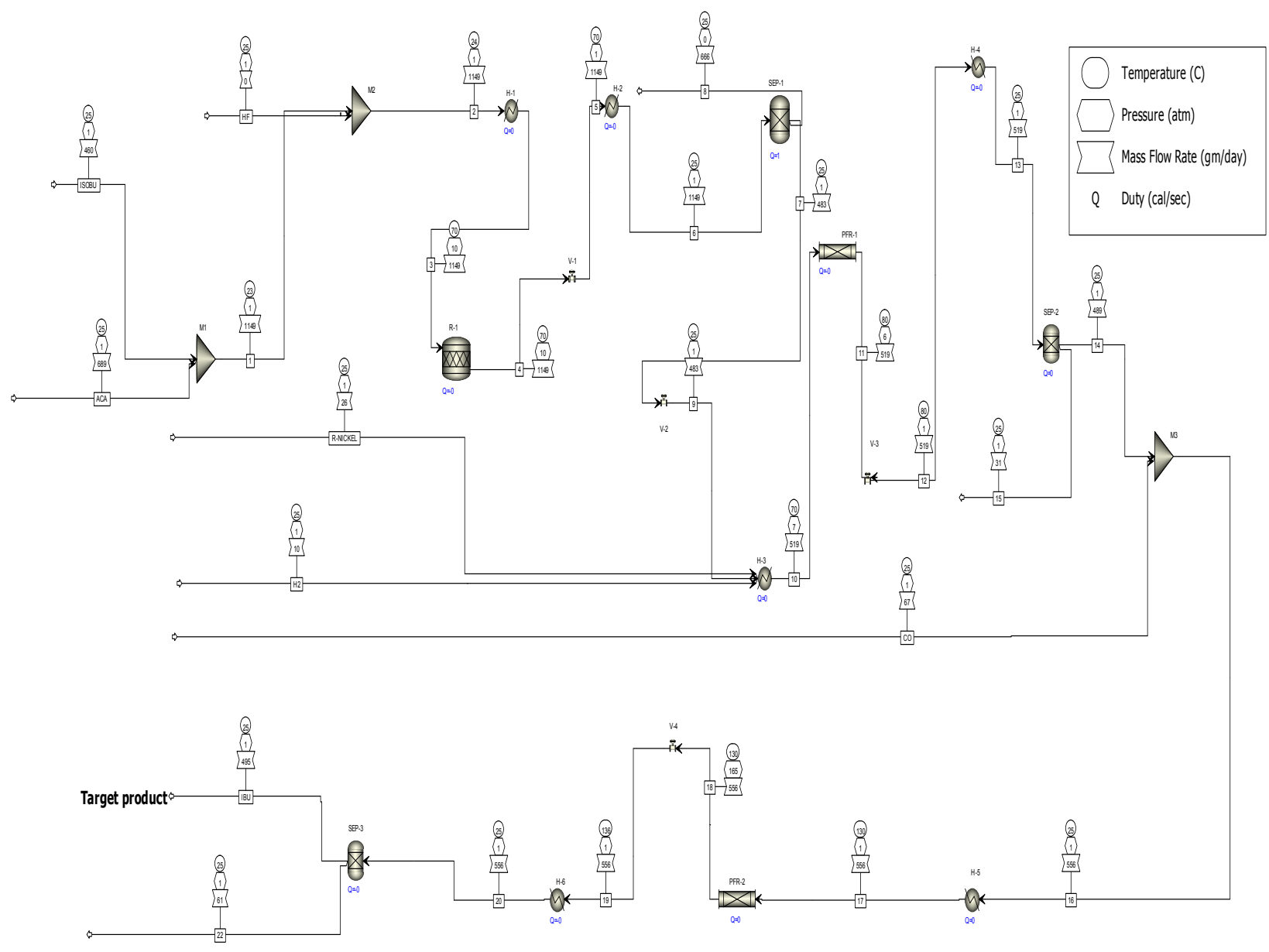

Table S6. Heat duty of the main equipment for the BHC route

\begin{tabular}{lll}
\hline Equipment & Code & Heat duty, kW \\
\hline Heat exchanger 1 & H1 & 0.001076 \\
Heat exchanger 2 & H2 & 0.001076 \\
Heat exchanger 3 & H3 & 0.000528 \\
Heat exchanger 4 & H4 & 0.000674 \\
Heat exchanger 5 & H5 & 0.001347 \\
Heat exchanger 6 & H6 & 0.00143 \\
Flow reactor 1 & R-1 & 0.001604 \\
Flow reactor 2 & R-2 & 0.002083 \\
Flow reactor 3 & R-3 & 0.001036 \\
Separation column 1 & SEP-1 & 0.003885 \\
Separation column 2 & SEP-2 & 0.000019 \\
Separation column 3 & SEP-3 & 0.000016 \\
\hline
\end{tabular}


E2. Bogdan synthetic route

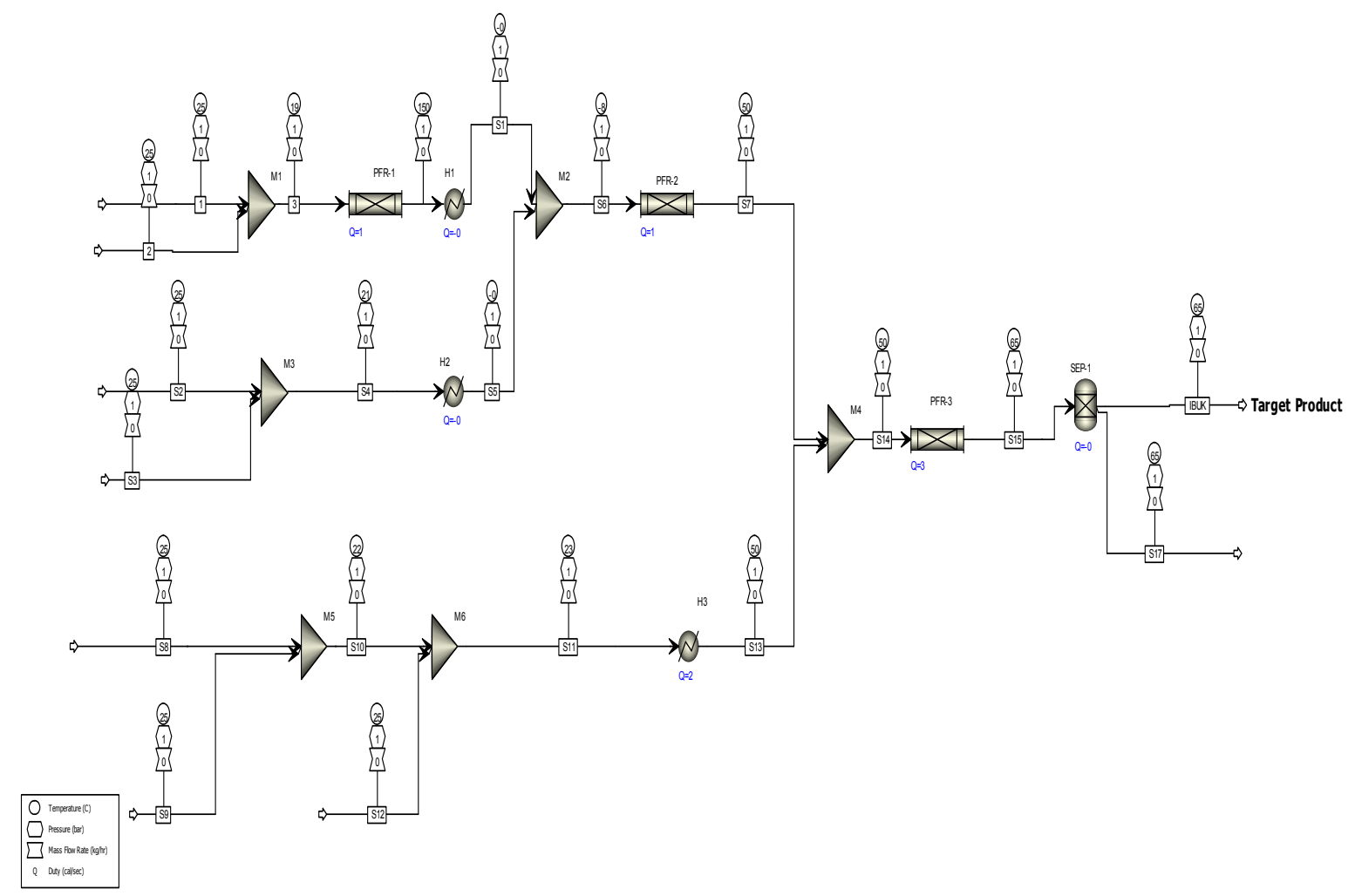

Table S7. Heat duty of the main equipment for the Bogdan synthetic route

\begin{tabular}{lll}
\hline Equipment & Code & Heat duty, kW \\
\hline Heat exchanger 1 & H1 & -0.002038203 \\
Heat exchanger 2 & H2 & -0.001570048 \\
Heat exchanger 3 & E3H3 & 0.008547978 \\
Flow reactor 1 & PFR-1 & 0.004509297 \\
Flow reactor 2 & PFR-2 & 0.004722757 \\
Flow reactor 3 & PFR-3 & 0.011492787 \\
Separation column 1 & SEP-1 & -0.00093496 \\
\hline
\end{tabular}




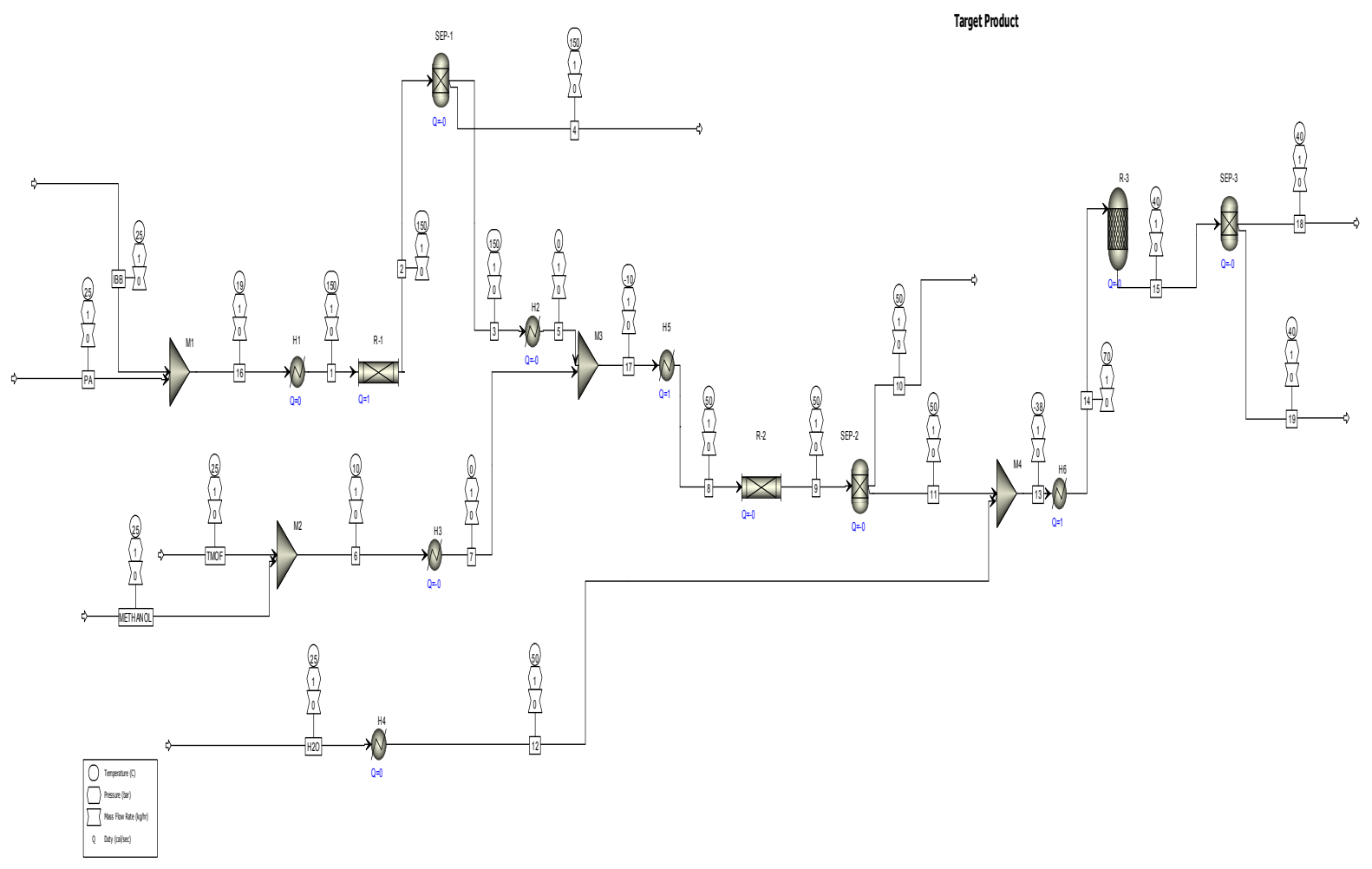

Table S8. Heat duty of the main equipment for the Bogdan synthetic route

\begin{tabular}{lll}
\hline Equipment & Code & Heat duty, kW \\
\hline Heat exchanger 1 & H1 & 0.001885336 \\
Heat exchanger 2 & H2 & -0.001660049 \\
Heat exchanger 3 & H3 & -0.000633844 \\
Heat exchanger 4 & H4 & 0.000219705 \\
Heat exchanger 5 & H5 & 0.004226038 \\
Heat exchanger 6 & H6 & 0.004856786 \\
Flow reactor 1 & PFR-1 & 0.004477326 \\
Flow reactor 2 & PFR-2 & $-2.63 \mathrm{E}-05$ \\
Flow reactor 3 & PFR-3 & -0.001488754 \\
Separation column 1 & SEP-1 & $-7.31 \mathrm{E}-05$ \\
Separation column 2 & SEP-2 & -0.000470412 \\
Separation column 3 & SEP-3 & -0.000164514 \\
\hline
\end{tabular}

